\title{
Ensayo
}

\section{La Comisión Científica Permanente y La Academia Nacional de Colombia ${ }^{1}$}

\section{Santiago Díaz-Piedrahita}

Un primer intento por revivir los ideales de la Expedición Botánica ocurrió durante la primera gestión del general Francisco de Paula Santander; entonces se conformó la Comisión Científica Permanente, más conocida como Misión Zea, la cual había sido creada mediante decreto del 22 de julio de 1823 y protocolizada mediante decreto del 28 de julio de ese año. Esta empresa debía trabajar en armonía con la recién creada Universidad Central. Para lograr una verdadera eficiencia, los centros universitarios debían contar con el apoyo de institutos de investigación y para cumplir ese propósito se buscó estimular el desarrollo científico retomando los ideales de la Expedición Botánica; para ello se puso en marcha la Misión que tenía como finalidad la de contratar en Europa varios científicos para que estableciesen en el país un museo de ciencias naturales y una escuela de minas; paralelamente debían organizar las cátedras de mineralogía, geología, química general, química aplicada, anatomía comparada, zoología, botánica, agricultura, dibujo, matemáticas, física y astronomía. La Misión estuvo integrada por cuatro naturalistas franceses y por uno peruano con formación europea. Se trataba del químico Jean-Baptiste Boussingault, quien impartiría las cátedras de química y mineralogía; del médico y naturalista François-Desiré Roulin, responsable de las cátedras de fisiología y anatomía comparada; de los auxiliares Jacques Bourdon y [Justin]-Marie Goudot, preparadores y taxidermistas entrenados en el Museo de Historia Natural de París, y del también químico e ingeniero de minas Mariano Rivero, futuro director de la Escuela de Minas y encargado de la organización del museo.

Los contratos que preveían la conformación de una biblioteca especializada en ciencias naturales, la adquisición de los instrumentos necesarios para adelantar las tareas docentes y de investigación, y la dotación del laboratorio de química, fueron aprobados en mayo de 1822. El grupo de científicos llegó al país hacia finales del año, pero debido a las trabas burocráticas sus integrantes sólo iniciaron las tareas propias de su profesión y del compromiso contraído varios meses después. A causa del incumplimiento del gobierno, que apenas firmó el decreto pertinente en 1823, estos científicos terminaron dedicándose a otras actividades con el fin de sobrevivir. Por desidia administrativa se perdió la oportunidad de que tanto en los laboratorios como en las aulas de la Universidad Central se formara académicamente una generación de estudiosos bajo la tutela de catedráticos idóneos. La universidad y el museo lograron un desarrollo incipiente que se vio interrumpido por la agitación política y por los hechos de septiembre de 1828 .

En 1826 se estableció la Academia Nacional de Colombia, un cuerpo integrado por 21 personalidades de Colombia y Venezuela. De acuerdo con las normas prescritas, eran 8. La nómina correspondiente aparece en la Gaceta de Colombia No. 266 de 1826. Las funciones de la Academia eran las de establecer, fomentar y propagar el conocimiento y perfección de las artes, de las letras, de las ciencias naturales y exactas y de la moral y de la política. ${ }^{2}$ El acto de instalación se realizó el 25 de diciembre, en presencia de

\footnotetext{
${ }^{1}$ Tomado de: Díaz-Piedrahita, Santiago. "Antecedentes de la Academia Colombiana de Ciencias Exactas, Físicas y Naturales”. En: Forero González, Enrique y Santiago Díaz-Piedrahita (eds.). La Academia Colombiana de Ciencias Exactas, Físicas y Naturales en los albores del siglo XXI. Bogotá: Academia Colombiana de Ciencias Exactas, Físicas y Naturales, 2012, Volumen I, pp. 18-21.

${ }^{2}$ En líneas generales, estos objetivos son los que cumplen en la actualidad las distintas entidades que
} 
José Manuel Restrepo quien ocupaba la Secretaría del Interior. La Academia fue presidida por Félix Restrepo, y como secretario actuó Pedro Acevedo Tejada. La vida de esta corporación resultó efímera. La rebelión encabezada por José Antonio Páez en Venezuela, la inoportunidad de proponer una Constitución Bolivariana o de crear una Confederación Andina, la propuesta de modificar la Constitución de Cúcuta antes del plazo estipulado en sus cláusulas, la conspiración septembrina, las presiones políticas y los demás hechos que, en 1830, desembocaron en la disolución de la Gran Colombia, dividieron la opinión y, como resulta obvio, no fueron propicios para el desarrollo de una Academia. A pesar de haber emprendido una transformación política y administrativa, la primera administración de Santander terminó en medio de una crisis política.

Además de la inestabilidad política, de las rivalidades regionales y de la falta de recursos económicos, fue la carencia de una comunidad intelectual y científica madura, la que impidió el desarrollo de la Academia Nacional. Por su parte la Universidad no alcanzó el desarrollo esperado y la educación superior siguió a cargo de los Colegios Mayores, en tanto que la Comisión Científica no produjo los frutos esperados.

Disuelta la Gran Colombia, se reorganizó el país bajo el nombre de República de la Nueva Granada y, una vez más bajo los auspicios de Santander, quien había asumido la presidencia el 15 de noviembre de 1832, se estableció por decreto la Academia Nacional de la Nueva Granada. La instalación se produjo el 6 de enero de 1833; el acto correspondiente se celebró en el propio Palacio de Gobierno bajo la presidencia del general y con la asistencia de catorce de sus veintiún integrantes.

El articulado de la norma constitutiva señala a la letra:

Artículo $1^{\circ}$. Se establece la Academia Nacional de la Nueva Granada con veintiún miembros, en los términos prescritos por la ley de 18 de marzo de 1826 y para los objetos que ella determina. Artículo $2^{\circ}$. Se nombrarán miembros de la Academia Nacional:

$1^{\circ}$ A los granadinos que pertenecían a la Academia Nacional de Colombia, a saber: los señores Vicente Azuero, José Manuel Restrepo, Manuel Benito Rebollo, José María del Castillo, Francisco Soto, Jerónimo Torres, Santiago Arroyo, Pedro Gual, Francisco Vergara y Benedicto Domínguez.

$2^{\circ}$ A los señores Joaquín Mosquera, Diego Fernando Gómez, Rufino Cuervo, Joaquín Acosta, reverendo obispo de Santa Marta (José María Estévez), Joaquín García, Lino de Pombo, Manuel María Quijano, Juan María Céspedes, General Hilario López y José María Triana. ${ }^{3}$

Entre otras ideas, el General Santander señaló durante el acto de instalación:

Señores,

La Patria os llama por el órgano del Gobierno a componer la Academia Nacional de la Nueva Granada. Vuestra comisión se contrae a establecer el imperio de la verdad en esta naciente República, afianzarlo y conservarlo perpetuamente.

Vuestras luces y vuestro patriotismo me inspiran la más fundada esperanza de que os dedicareis asiduamente a corresponder el bello encargo que la Patria os confía. Ya que somos tan dichosos de que algunos de nuestros primeros guías en la carrera de la Independencia y de la Libertad escapasen del estrago general causado por la cuchilla española, y de que la discordia civil respetase los preciosos renuevos que 22 años de sacrificios han fecundado, debemos marchar con el siglo, concurriendo a comunicar a nuestros compatriotas las verdades útiles, que hacen desarrollar la civilización intelectual, industrial y política. ${ }^{4}$

La vida de esta institución, como la de su predecesora, fue fugaz; paulatinamente cayó en la inoperancia por la falta de compromiso y desinterés de sus integrantes. La inestabilidad política afectó no sólo a la Academia sino a la Universidad. El anhelado desarrollo científico y cultural seguía sin alcanzarse.

integran el Colegio Máximo de las Academias de Colombia, organismo creado en 1959.

${ }^{3}$ López Domínguez, Luís Horacio (comp.). Obra educativa de Santander: 1827-1835. Bogotá: Biblioteca de la Presidencia de la República, 1990, Tomo II, pp. 87-88.

${ }^{4}$ Ibidem, pp 104-105. 
Con la organización de la Academia Nacional se trató, infructuosamente, de dar vida y dinamismo a los gabinetes de historia natural, al Museo Nacional y al Observatorio Astronómico, entidades que debían de servir de apoyo a la Universidad y a la propia Academia. Santander había procurado modernizar la enseñanza y reducir el poder de la Iglesia Católica en el terreno educativo. Entre 1839 y 1841 se produjo la llamada Guerra de los Supremos, y el gobierno presidido por Pedro Alcántara Herrán dio un claro viraje hacia la derecha, cambio ideológico defendido arduamente por Mariano Ospina Rodríguez, quien se desempeñaba como Secretario del Interior. En 1844 Ospina invitó a los jesuitas a regresar al país y les solicitó reasumiesen las tareas docentes. En forma paralela, la Universidad Central fue desarticulada para dar paso a tres pequeñas universidades de distrito fácilmente manejables. A Bogotá le correspondió la Universidad del Primer Distrito, donde al lado de una estricta disciplina imperaba una ideología claramente conservadora y religiosa que poco favorecía el desarrollo de las ciencias básicas. Muchas asignaturas de los programas fueron suprimidas por considerarse peligrosas en la formación de la juventud.

Don Mariano trató de compaginar su ideología con el fomento de las ciencias útiles, un tema que le preocupaba. ${ }^{5}$ Para él, como buen antioqueño, en el país imperaba una sociedad eminentemente agraria que se hallaba saturada de abogados, literatos, teólogos y generales, motivo por el cual los jóvenes requerían nuevos horizontes; esa apertura a los campos empresarial e industrial y minero se lograría través de una educación más técnica y en campos más prácticos. Su plan de estudios buscaba formar hombres útiles y disminuir el número de abogados que, a su juicio, era excesivo, carecía de empleo y entrababa la marcha política de la Nación. Por ello hacía énfasis en las matemáticas y las ciencias naturales, cuyo estudio debía anteceder al de la jurisprudencia. Pese a los esfuerzos de sus organizadores, la Universidad siguió difundiendo un saber de tipo escolástico sin llegarse a generar verdadera ciencia. Curiosamente, en este ambiente académico aparentemente adverso, se formó una generación de jóvenes como Manuel Murillo Toro, Santos Gutiérrez, Eustorgio Salgar y Francisco Javier Zaldúa, entre otros, que cambiaría temporalmente al país al implantar políticas radicales totalmente opuestas a las que habían aprendido en las aulas.

\footnotetext{
${ }^{5} \mathrm{Al}$ respecto puede verse Safford, Frank. El ideal de lo práctico. El desafio de formar una elite técnica y empresarial en Colombia. Bogotá: Universidad Nacional - El Áncora Editores, 1989.
} 